\title{
Seroprevalence of canine herpesvirus in breeding kennels in the Gauteng Province of South Africa
}

\author{
J.O. Nöthling ${ }^{a}$, D. Hüssy ${ }^{b}$, D. Steckler ${ }^{a}$ and M. Ackermann ${ }^{b}$
}

aSection of Reproduction, Department of Production Animal Studies, Faculty of Veterinary Science, University of Pretoria, South Africa

${ }^{\mathrm{b}}$ Institute of Virology, Vetsuisse Faculty, University of Zurich, Zurich, Switzerland

\begin{abstract}
Canine herpesvirus (CHV-1) causes neonatal deaths as well as infertility due to embryonal death, abortion and stillbirths in breeding kennels. The aim of this study was to determine the prevalence of antibodies against canine herpesvirus in the serum of dogs older than 1 year in breeding kennels in the Gauteng Province of South Africa. A serum neutralization test (SNT) and a newly developed enzyme linked immunosorbent assay (ELISA) were used to test the serum samples of 328 dogs in 38 breeding kennels. With SNT as well as ELISA, $22 \%$ of sera were positive $(P>0.9)$. Seventeen kennels $(45 \%$ of total kennels) each had at least one positive dog on SNT compared with twenty kennels ( $53 \%$ of total kennels) that each had at least one positive dog on ELISA $(P=0.6)$. The prevalence of positive dogs in positive kennels was $42 \pm 26 \%$ ( $n=17$ kennels) for SNT and $39 \pm 26 \%(n=20$ kennels) for ELISA. Pairwise comparison of kennels showed that the prevalence of SNT positive dogs was similar to the prevalence of ELISA positive dogs ( $P=0.3, n=38$ kennels). Seroprevalence was independent of age, gender or colony size. This study suggests that canine herpesvirus is sufficiently common in breeding dogs in the Gauteng Province of South Africa to pose a threat to neonatal survival and fertility.
\end{abstract}

Keywords: Canine herpesvirus; CHV-1; Serum neutralization test; ELISA; Dog; South Africa

\section{Introduction}

About 40 years ago, Carmichael et al. [1] described a highly fatal viral disease causing widespread focal necrosis in parenchymatous organs of newborn puppies up to 2 weeks old, which was subsequently identified as a herpesvirus [2]. Other reports on canine herpesvirus (CHV-1) soon followed [3], [4] and [5]. CHV-1 is of low pathogenicity in puppies older than 5 weeks [6] and may cause tracheobronchitis in adult dogs [7]. Apart from the important disease in newborn puppies, CHV-1 also affects reproduction of dogs in other ways: the virus may cause vesicular lesions in the vestibulum and vagina of the bitch, as well as on the penis and the preputial mucosa of dogs [8] and may cause fetal death, abortion and stillbirths [8] and [9]. CHV-1 thus poses a serious disease to the reproductive efficiency of breeding kennels. The virus is transmitted oronasally [1], transplacentally [9] and venereally [8]. Lesions in the vestibulum and vagina of bitches may recur when bitches come into proestrus and regress when they go into anestrus [8]. Affected animals may remain latent carriers [10], and the virus was identified in the lumbosacral ganglia, tonsils, parotid salivary glands and liver of dogs that showed no sign of herpesvirus infection [11]. 
In an unvaccinated animal the presence of antibodies against CHV-1 indicates previous exposure to the virus. Evermann [12] states that titers of antibodies against CHV-1 may rise and fall within 4-8 weeks following exposure, although König et al. [13] showed that titers of serologically positive dogs remained the same over the 5-15 months separating two examinations.

Numerous epidemiological factors affect the prevalence of antibodies in the serum of dogs (seroprevalence). Bitches that had recently lost litters or were infertile have a higher seroprevalence than those with no such problems [14]. Although one study reported a similar seroprevalence in dogs kept in breeding kennels than in individually kept dogs [15], another suggests that dogs kept singly had a lower seroprevalance than dogs in kennels [16]. The seroprevalence is also higher in breeding kennels with a history of neonatal deaths than those without [14]. Dogs in larger kennels are more likely to have antibodies against the virus than those in smaller ones [14] and [17], especially if the hygiene in the larger kennels is poor [17]. Antibody titers are lower in younger bitches than in older ones [17]. Male dogs that have mated are also more likely to have antibodies than those that have never mated [17].

Carmichael et al. [18] showed that CHV-1 is neutralized with low dilutions of dog serum and that a two- to eightfold increase in titers of antibodies occurs if complement is used in an SNT. ELISA tests seem more sensitive than SNT [15] and [19] although Reading and Field [20] reported an SNT that had similar sensitivity to the ELISA they used.

$\mathrm{CHV}-1$ is present in many countries and the prevalence of antibodies against CHV-1 varies from $6 \%$ in some countries to over $90 \%$ in others. In one study reported in $1974,6 \%$ of dogs from a variety of sources in Washington were seropositive by an SNT without complement [21]. Similarly, in a study in Switzerland in which an SNT with complement was used, only $6 \%$ of individually reared dogs were seropositive [16]. In the latter study [16] 134 dogs from 13 breeding kennels with reproductive problems were tested and the percentages of dogs, kennels and dogs in positive kennels that were positive were $18.7 \%, 23.1 \%$ and $36.8 \%$, respectively. A study done in Germany and reported in 1976 (using an SNT with complement) showed that $22 \%$ of tested dogs, $39 \%$ of dogs from kennels with problems with puppy deaths and $40 \%$ of kennels with such problems, were positive [22]. More recently (1997-1998), a study in which an ELISA was used showed that the seroprevalence in dogs in the Netherlands was about $40 \%$ [23] whereas, at about the same time, $94 \%$ of pet dogs in England were positive on either ELISA or SNT with complement [24]. Having used an SNT with complement, Van Gucht et al. [14] investigated 18 Belgian breeding kennels and found that $50 \%$ of dogs and $50 \%$ of kennels were positive. Another Belgian study, in which an ELISA was used, showed a seroprevalence of $46 \%$ for breeding dogs as well as nonbreeding dogs [15]. In a recent study in Germany on dogs kept in kennels it was shown that $18.8 \%$ of all dogs and $65.2 \%$ of dogs from positive kennels were serologically positive, while $26 \%$ of kennels had serologically positive dogs [13].

The seroprevalence of $\mathrm{CHV}-1$ in South Africa is unknown. South Africa has nine provinces. Approximately half the breeders and $48 \%$ of all dogs registered with the Kennel Union of South Africa (KUSA), which is the largest registering body in South Africa, are from the Gauteng province (M. Darwin, KUSA, 2004, personal communication). At the time that this study was planned no vaccine against CHV-1 was available in South Africa, thus the 
serological status of the South African dog population had not yet been affected by vaccination. A pharmaceutical company was, however, planning to introduce a vaccine in South Africa for use on breeding bitches. Considering that canine herpesvirus is of greatest economical importance in breeding kennels, it was decided to determine the seroprevalence of CHV-1 in breeding kennels in the Gauteng Province of South Africa before the onset of vaccination.

\section{Materials and methods}

\subsection{Dogs}

All the names of breeding kennels in Gauteng from which dogs were presented during the past 3 years to the Small Animal Reproduction Clinic of the Onderstepoort Veterinary Academic Hospital, or to either of two private specialists in canine reproduction, irrespective of the number of dogs presented and the reason why they were presented, were put on a list. To each of the 104 kennels in this list a unique random number between 1 and 10,000 was assigned, and the kennels subsequently ordered according to the size of the random number. From the sorted list the first 38 kennels that had at least four dogs older than 1 year and where the owners agreed to participate in the study were used. This method of selecting the participating kennels is considered justified because collectively the two practicing veterinarians and the academic clinic provide service to a large number of dog breeders in Gauteng and their collective client bases may be assumed to be representative of dedicated breeders in Gauteng.

Ten milliliters of blood were collected from each dog older than 1 year in each participating kennel. All blood samples were collected within a period of 2 weeks. The serum was separated and stored at $-18{ }^{\circ} \mathrm{C}$ until the last serum sample had been frozen, subsequent to which the frozen samples were packed in an insulated container with solid carbon dioxide at $-78{ }^{\circ} \mathrm{C}$ and sent from South Africa to the University of Zurich, Switzerland, where the samples were received 4 days later in a still frozen state and kept frozen at $-80{ }^{\circ} \mathrm{C}$ until the analyses were done 47-60 days after arrival in Switzerland.

Each serum sample was evaluated by means of an SNT and thereafter by means of an ELISA test.

\subsection{Media, cell cultures and virus strain}

Cell growth medium consisted of minimal essential medium (MEM) buffered with $25 \mathrm{mM}$ HEPES (Amimed, Biocept, Allschwil, Switzerland), supplemented with $7 \%(\mathrm{v} / \mathrm{v})$ foetal calf serum (FCS; Omnilab, Mettmenstetten, Switzerland) and antibiotics (100 units/ml penicillin and $0.1 \mathrm{mg} / \mathrm{ml}$ streptomycin; Sigma-Aldrich, Buchs, Switzerland). Cell maintenance medium was identical but with FCS reduced to $2 \%$. Virus infection medium was essentially FCS-free and consisted of MEM with 25 mM HEPES.

MDCK cells at passages 221-226 (ATCC) and a CHV-1 field strain [25] with a titer of $10^{7.5} \mathrm{TCID}_{50} \mathrm{ml}^{-1}$ were used. 


\subsection{Serum neutralization test (SNT)}

The method of Engels et al. [16] was used, except that no complement was used. Briefly, the sera were inactivated in a heatblock at $56{ }^{\circ} \mathrm{C}$ for 30 min followed by a centrifugation step at $13,000 \mathrm{rpm}$ for $10 \mathrm{~min}$ in a microcentrifuge. Subsequently, $250 \mu \mathrm{l}$ of CHV-1 stock previously diluted in cell maintenance medium to provide $2000 \mathrm{TCID}_{50} \mathrm{ml}^{-1}$ were added to $250 \mu \mathrm{l}$ serum and incubated for $2 \mathrm{~h}$ at $37^{\circ} \mathrm{C}$. Subsequently, $100 \mu \mathrm{l}$ of this mixture were transferred into wells of a microtiter plate containing MDCK cell monolayers (15,000 cells per well, grown overnight). Four wells were used per sample. Control of virus titer, as well as serum and cell controls was included in each test series.

After an incubation period of 3 days at $37^{\circ} \mathrm{C}$, the plate was read microscopically. A serum sample was considered to be positive if no cytopathic effect was visible in at least one of its four test wells.

A random sample of eight sera that were SNT positive were further analyzed in twofold dilutions to determine the antibody titers.

\subsection{ELISA}

\subsubsection{Production of ELISA antigens}

ELISA-antigen was prepared as previously described for EHV-1 and PEDV [26] and [27].

Briefly, six $75 \mathrm{~cm}^{2}$ flasks of 1-day-old monolayers, each containing $5.2 \times 10^{6}$ of MDCK cells, were washed three times with phosphate-buffered saline (PBS). Three of them were infected: each was overlaid with $2 \mathrm{ml}$ infection medium containing $\mathrm{CHV}-1$ at a multiplicity of infection of 10 . The remaining three flasks were mock-infected with virus infection medium.

After an adsorption period of $2 \mathrm{~h}$ at $37^{\circ} \mathrm{C}$, the monolayers were washed twice with virus infection medium before $13 \mathrm{ml}$ of virus infection medium were added to each flask for further incubation at $37^{\circ} \mathrm{C}$.

After $24 \mathrm{~h}$, the virus-specific cytopathic effect reached $100 \%$ and the cells were harvested by scraping. The cells of the three virus-infected flasks were pooled as well as those of the three mock-infected flasks. All of the following steps were performed on ice or at $4{ }^{\circ} \mathrm{C}$. Cells and supernatant were centrifuged at $311 \times g$ for $10 \mathrm{~min}$ at $4{ }^{\circ} \mathrm{C}$. The cells were washed once with PBS: the pellet was resuspended in $50 \mathrm{ml}$ PBS and centrifuged (at $311 \times \mathrm{g}, 10 \mathrm{~min}$, $4{ }^{\circ} \mathrm{C}$ ). The cells were resuspended in $900 \mu$ of double-distilled water and put on ice. After $10 \mathrm{~min}, 100 \mu \mathrm{l}$ 0.15\% (w/v) OBG ( $n$-octyl-beta-d-glucopyranoside; Sigma-Aldrich) was added in order to disrupt the membranes of the cells. After applying $25 \mu \mathrm{l} 1 \mathrm{M} \mathrm{Tris,} \mathrm{pH} 7.5$, the sample was kept on ice for 10 min before being pelleted in Eppendorf tubes at $16,060 \times g$ for $15 \mathrm{~min}$ at $4{ }^{\circ} \mathrm{C}$ in order to separate the soluble fractions from the insoluble components. The soluble fraction from the lysate was supplemented with $3 \mu \mathrm{l}$ of $1 \mathrm{mM}$ PMFS (phenylmethylsulfonyl fluoride; Sigma-Aldrich). Aliquots of $125 \mu \mathrm{l}$ were stored at $-20{ }^{\circ} \mathrm{C}$ until further use. 


\subsubsection{Performing the ELISA tests}

ELISA plates (Nunc-Immuno ${ }^{\mathrm{TM}} 96$ MicroWell $^{\mathrm{TM}}$ Plates, MaxiSorp ${ }^{\mathrm{TM}}$; Milian, Geneva, Switzerland) were sensitized with ELISA antigens diluted 1:100 in coating buffer $(0.05 \mathrm{M}$ sodium bicarbonate, $\mathrm{pH}$ 9.7). Rows of wells on the plates were alternately coated with $200 \mu \mathrm{l}$ of diluted virus antigen or mock antigen per well and kept overnight at $4{ }^{\circ} \mathrm{C}$. The plates were washed four times with PBS supplemented with $0.05 \%$ Tween 20 and four times with double-distilled water.

Test sera were diluted 1:100 with PBS-Tween and $200 \mu \mathrm{l}$ of each serum dilution were added to both a virus antigen coated and a mock antigen coated well. After an incubation period of 90 min at room temperature, the plate was washed four times with PBS-Tween. Then, $200 \mu \mathrm{l}$ aliquots of horseradish peroxidase-conjugated anti-dog secondary antibody (SigmaAldrich), diluted 1:10,000 in PBS-Tween, were pipetted to each well and the plate was incubated for $90 \mathrm{~min}$ at room temperature. After repeating the four PBS-Tween washes, $200 \mu \mathrm{l}$ of substrate solution $\left(78 \mathrm{mM}\right.$ acetic acid, $24 \mathrm{mM} \mathrm{CH}_{3} \mathrm{COONa}_{3} 3 \mathrm{H}_{2} \mathrm{O}, 50 \mathrm{mM} \mathrm{NaH}_{2} \mathrm{PO}_{4}$. $1 \mathrm{H}_{2} \mathrm{O}, 2 \mathrm{mM}$ ABTS [Roche, Rotkreuz, Switzerland] and $1.25 \mathrm{mM} \mathrm{H}_{2} \mathrm{O}_{2}$ applied shortly before use) was added. The absorption at $405 \mathrm{~nm}$ was measured after 30 and $40 \mathrm{~min}$ in an Anthos reader 2001 (Anthos Labtecs Instruments $\mathrm{GmbH}$, Wals, Austria). For each serum sample, the difference in optical density between the well containing virus and mock antigen was determined after $30 \mathrm{~min}$ (dOD30) and after 40 min (dOD40).

\subsection{Statistical analysis}

Two-way comparisons of numerical data were done by means of a $t$-test with equal or unequal variance, or a Wilcoxon Rank-sum test, depending on the distribution of the data [28]. Two-way comparisons of proportions were done by means of a Chi-square test if all cell counts exceeded five, otherwise Fischer's Exact probability test was used [29]. The percentages of positive animals on SNT and ELISA in each kennel were compared by means of a paired $t$-test, after Bartlett's adjustment of percentages of zero or 100, and arcsine transformation of each percentage [28]. The Pearson correlation procedure was used to determine the correlation between two normally distributed variables and the Spearman rank correlation procedure if the variables were not normally distributed [28]. A kappa test was used to determine the level of agreement between the SNT and ELISA tests [30]. Variation is shown as mean \pm standard deviation. The NCSS software package (Kaysville, UT, USA) was used for the statistical analyses.

\section{Results}

The 328 dogs were from 38 kennels from various parts of Gauteng. Twenty-nine breeds were represented. The number of dogs per kennel that were older than 1 year varied from 3 (in 1 kennel) to 20 , with an average of $8.6 \pm 4.2$. The mean age of the 328 dogs was $4.2 \pm 2.6$ years and they consisted of 109 males and 219 females. 
Of the 328 samples, 68 were positive on SNT, 247 were negative and 13 could not be interpreted by SNT as they were cytotoxic. The eight SNT positive sera, in which titers were determined, had low titers of 1:2-1:16.

When the results of the 315 sera that were suitable for SNT were ordered according to reactions at 30 min after addition of the substrate (dOD30), it was found that all but five with dOD30 $<0.2$ were SNT negative and all but two with dOD30 $\geq 0.2$ were SNT positive. When the same 315 sera were ordered according to dOD40, all but two with dOD40 $<0.2$ were SNT negative and all but three with dOD40 $\geq 0.2$ were SNT positive. Furthermore, the median of the difference between dOD40 and dOD30 for sera that were SNT positive was 0.096 compared to 0.003 for sera that were SNT negative $(P<0.001)$, suggesting that dOD40 discriminates more clearly between SNT negative and SNT positive samples than dOD30. Hence, dOD40 and a cut-off of 0.2 were used to classify sera.

Using the SNT as gold standard, the sensitivity of the ELISA was $97.1 \%$ and the specificity $98.8 \%$. Cytotoxic sera were not taken into account.

Sixty-six of the 68 SNT positive samples plus another three SNT negative samples and two samples that were cytotoxic reacted positive on ELISA, giving 71 ELISA positive samples. Thus, $21.5 \%$ of dogs older than 1 year were SNT positive (excluding the cytotoxic sera samples) and $21.6 \%$ were positive on ELISA. The proportions of dogs that were positive were similar for SNT and ELISA $(P>0.9)$. The value of kappa was 0.95 , suggesting an almost perfect agreement between the results of the SNT and the ELISA [30].

Seventeen kennels had at least 1 positive dog on SNT whereas the same 17 had positive dogs on ELISA and another 3 each had 1 ELISA positive dog, giving $45 \%$ positive kennels on SNT and $53 \%$ on ELISA. The proportions of kennels that were positive, were similar for SNT and ELISA $(P=0.6)$.

Six kennels could not be classified as either positive or negative on SNT because each had some cytotoxic sera while the remainder of the sera were SNT negative. Of these six kennels, one had a single positive value on ELISA.

For SNT, the prevalence in positive kennels varied from $10 \%$ to $88 \%$ with a mean of $42 \%$ and a standard deviation of $26 \%(n=17)$. On ELISA, the prevalence in positive kennels varied from $10 \%$ to $100 \%$, with a mean of $39 \%$ and a standard deviation of $26 \%(n=20)$. The prevalences in kennels of dogs that were positive on SNT or ELISA did not differ $(P=0.3, n=38$ kennels $)$.

SNT positive kennels had $5-18(9.2 \pm 3.8)$ dogs older than 1 year, whereas negative kennels had 3-20 (8.2 \pm 4.5$)$. ELISA positive kennels had 3-18 $(8.5 \pm 3.9)$ dogs older than 1 year, whereas negative kennels had 4-20 $(8.8 \pm 4.6)$. For SNT as well as ELISA, the size of positive kennels was similar to that of negative kennels $(P>0.8)$.

There was no correlation between kennel size and seroprevalence rate, irrespective of whether all 38 kennels were included in the analysis (Spearman's rank correlation coefficient $0.1, P=0.6$ ) or whether only the 20 kennels that were positive on ELISA were considered 
(Spearman's rank correlation coefficient $0.2, P=0.4$ ) or when the 17 kennels that were positive on SNT were considered (Spearman's rank correlation coefficient $0.1, P=0.7$ ).

In positive kennels, the median age of the 99 dogs that were ELISA negative was 4 years, which was the same as that of the 71 ELISA positive dogs $(P=0.8)$. Furthermore, within the 20 positive kennels, dOD40 of bitches was not correlated to age, irrespective of whether all 117 bitches in those kennels were included in the analysis or only the 52 serologically positive ones $(P \geq 0.5)$. In the same way, dOD40 was not correlated to age for the 53 males or 19 positive males in the 20 positive kennels $(P=0.4)$.

Serological status was independent of gender, as $17.4 \%$ of the 109 males and $23.8 \%$ of the 219 females were ELISA positive $(P=0.2)$.

Dogs of 14 breeds were positive on SNT, whereas dogs of 15 breeds were positive on ELISA. Among those three positive kennels that had at least two positive animals and kept at least two breeds, infection occurred in representatives of all three breeds in one and in three of the four breeds in another, whereas both positive animals from one kennel with four breeds were of the same breed.

Of the ELISA positive kennels, seven had only one positive dog and 13 had more than one. The seven kennels were smaller than the other 13 (5.9 \pm 2.2 vs. $9.9 \pm 3.9$ animals older than 1 year, $P=0.01)$. Dogs in the seven kennels were younger than those in the other 13 (3.3 \pm 1.3 years, $n=41$ vs. $5.1 \pm 3.4$ years, $n=129$; $P<0.001$ ). In the seven kennels, positive dogs were of the same age and gender ratio as negative dogs $(2.7 \pm 0.8$ years and $29 \%$ males for the seven positive dogs vs. $3.5 \pm 1.4$ years and $41 \%$ males for the 34 for negative dogs, $P \geq 0.2$ ).

\section{Discussion}

In Gauteng, where about half of South Africa's breeding dogs occur, the seroprevalence for $\mathrm{CHV}-1$ was $53 \%$ for breeding colonies and $22 \%$ for breeding dogs older than 1 year.

Carmichael et al. [18] reported that neutralisation of CHV-1 without the use of complement only occurred if low serum dilutions were used. In the SNT of the current study no complement was used. It seems unlikely the fact that no complement was used in the current study affected the prevalence of SNT positive dogs, because the virus suspension was added to undiluted serum. Furthermore, at the institute where the serological analyses of the current study were performed, it was found that the addition of complement makes no or little difference to the outcome of the SNT. In support of the latter view, there are numerous mechanisms whereby antibodies can neutralise virus without the intervention of complement [31].

In contrast to other studies suggesting that ELISA is more sensitive than SNT [15] and [19], this study shows that, at the dog level as well as the level of kennels, the seroprevalences obtained with SNT and ELISA were similar, which is in agreement with the findings of Reading and Field [20]. In the current study there was an almost perfect agreement between the results obtained by the SNT and the ELISA tests. Using the SNT as gold standard, the sensitivity of the ELISA was $97.1 \%$ and the specificity $98.8 \%$. The specificity of the ELISA is 
probably underestimated as the SNT detects only neutralizing antibodies whereas the ELISA is able to detect also other specific but not neutralizing antibodies. These results suggest that the newly developed ELISA may be a good alternative tool to the labor-intensive and time-consuming SNT for sero-epidemiological surveys.

Apart from being a serious disease in breeding colonies, canine herpesvirus causes a mild but contagious upper respiratory infection [7]. In order to avoid a bias in serological status due to a seasonal exposure to respiratory infection, the minimum age of the subjects used in the current study was set at 1 year. This age restriction also avoided coincidentally testing more than one puppy from the same young litter as such littermates are biased towards a similar serological status because they had colostrum from the same source.

Ronsse et al. [17] reported a significant trend to higher titers of antibodies against canine herpesvirus with increasing age. Yet, in the 20 seropositive kennels of the current study, serological status was independent of age. In contrast to other studies showing that larger kennels are more likely to have positive dogs than smaller ones [14] and [17], the current study shows no correlation between colony size and seroprevalence. In harmony with the effects of age and colony size found by others [14] and [17], the current study does show that those kennels with only one positive dog were smaller and their dogs younger than kennels with more than one positive dog, possibly indicating that the infection spreads among dogs in a kennel as they remain together for longer.

Although the aim of the study was not to determine whether or not canine herpesvirus has a predisposition to certain breeds of dogs kept in Gauteng, the fact that approximately half the 29 breeds included in the study had positive animals suggests that a breed predisposition is unlikely.

This study suggests that the seroprevalence of canine herpesvirus in Gauteng is similar to the $18.7 \%$ in dogs from 13 breeding kennels in Switzerland [16] and the $19 \%$ of dogs from German kennels [13]. The proportion of positive kennels in the current study seems similar to the $50 \%$ of 18 breeding kennels in Belgium [14] but higher than the $26 \%$ of 412 kennels in Germany [13]. The seroprevalence in random samples of dogs in the Netherlands and England [23] and [24], respectively were significantly higher than that of dogs in Gauteng breeding kennels. Although Ronsse et al. [15] showed that individually kept dogs in Belgium had the same seroprevalence as dogs kept in kennels, Engels et al. [16], showed that the seroprevalence was higher in dogs kept in kennels, suggesting that the seroprevalence of $\mathrm{CHV}-1$ in Gauteng is lower than in the Netherlands or England. The average seroprevalence in positive kennels in Gauteng was 39\%, which appears lower than the $65-80 \%$ in other studies [13], [16] and [22]. From the above it appears that the seroprevalence in dogs in Gauteng breeding kennels are similar to those of dogs in Switzerland and Germany, but lower than those of dogs in the Netherlands and England, while the proportion of positive dogs in positive kennels is generally lower than those reported in Europe.

The current study suggests that CHV-1 is sufficiently common to pose a threat to neonatal survival and fertility in breeding kennels in Gauteng. 


\section{Acknowledgements}

We thank Dr. Monika Engels for technical advice and helpful discussions. This study was supported by a grant to M.A. from the University of Zürich.

\section{References}

[1] L.E. Carmichael, R.A. Squire and L. Krook, Clinical and pathologic features of a fatal viral disease of newborn pups, Am J Vet Res 26 (1965), pp. 803-814.

[2] J.D. Strandberg and L.E. Carmichael, Electron microscopy of a canine herpesvirus, $J$ Bacteriol 90 (1965), pp. 1790-1792.

[3] J. Prydie, M.J. Harrison and J. Graham, Isolation of a canine herpesvirus, Vet Rec 79 (1966), pp. 660-661.

[4] H.J.C. Cornwell, N.G. Wright, R.S.F. Campbell and R.J. Roberts, Neonatal disease in the dog associated with a herpes-like virus, Vet $\operatorname{Rec} 79$ (1966), pp. 661-662.

[5] H.J.C. Cornwell and N.G. Wright, Neonatal canine herpesvirus infection: a review of present knowledge, Vet Rec 84 (1969), pp. 2-6.

[6] M.J.G. Appel, M. Menegus, I.M. Parsonson and L.E. Carmichael, Pathogenesis of canine herpesvirus in specific-pathogen-free dogs: 5- to 12-week-old pups, Am J Vet Res 30 (1969), pp. 2067-2073.

[7] A. Karpas, F.G. Garcia, F. Calvo and R.E. Cross, Experimental production of canine tracheobronchitis (kennel cough) with canine herpesvirus isolated from naturally infected dogs, Am J Vet Res 29 (1968), pp. 1251-1257.

[8] G. Poste and N. King, Isolation of a herpesvirus from the canine genital tract: association with infertility, abortion and stillbirths, Vet Rec 88 (1971), pp. 229-233

[9] A. Hashimoto, K. Hirai, Y. Suzuki and Y. Fujimoto, Experimental transplacental transmission of canine herpesvirus in pregnant bitches during the second trimester of gestation, Am J Vet Res 44 (1983), pp. 610-614.

[10] Y. Okuda, K. Ishida, A. Hashimoto, T. Yamaguchi, H. Fukushi and K. Hirai et al., Virus reactivation in bitches with a medical history of herpesvirus infection, Am J Vet Res 54 (1993), pp. 551-554.

[11] P.D. Burr, M.E.M. Campbell, L. Nicolson and D.E. Onions, Detection of canine herpesvirus 1 in a wide range of tissues using the polymerase chain reaction, Vet Microbiol 53 (1996), pp. 227-237. 
[12] J.F. Evermann, Diagnosis of canine herpetic infections. In: R.W. Kirk, Editor, Current veterinary therapy, small animal practice X, WB Saunders, Philadelphia (1989), pp. 13131316.

[13] M. König, J. Neiseke and H.J. Thiel, Prevalence of canine herpesvirus 1 (CHV-1) in German kennels, Tierarztl Umsch 59 (2004), pp. 559-565.

[14] S. Van Gucht, H. Nauwynck and M. Pensaert, Prevalence of canine herpesvirus in kennels and the possible association with fertility problems and neonatal death, Vlaams Diergeneeskundig Tijdschrift 70 (2001), pp. 204-211.

[15] V. Ronsse, J. Verstegen, K. Onclin, A.L. Guiot, C. Aeberlé and H.J. Nauwynck et al., Seroprevalence of canine herpesvirus-1 in the Belgian dog population in 2000, Reprod Domest Anim 37 (2002), pp. 299-304.

[16] M. Engels, B. Mayr-Bibrack, B. Ruckstuhl, A. Metzler and R. Wyler, The seroepizootiology of canine herpes virus infections in Switzerland and preliminary studies with a vaccine, Zb/ Vet Med B 27 (1980), pp. 257-267

[17] V. Ronsse, J. Verstegen, K. Onclin, F. Farnir and H. Poulet, Risk factors and reproductive disorders asssociated with canine herpesvirus-1 (CHV-1), Theriogenology 61 (2004), pp. 619-636.

[18] L.E. Carmichael, Herpesvirus canis: aspects of pathogenesis and immune response, $J$ Am Vet Med Assoc 156 (1970), pp. 1714-1721.

[19] A. Takumi, K. Kusanagi, K. Tuchiya, X. Xuan, M. Azetaka and E. Takahashi, Serodiagnosis of canine herpesvirus infection-development of an enzyme-linked immunosorbent assay and its comparison with two improved methods of serum neutralization test, Jpn J Vet Sci 52 (1990), pp. 241-250.

[20] M.J. Reading and H.J. Field, Detection of high levels of canine herpes virus-1 neutralising antibody in kennel dogs using a novel serum neutralisation test, Res Vet Sci 66 (1999), pp. 273-275.

[21] R.W. Fulton, R.L. Ott, J.C. Duenwald and J.R. Gorham, Serum antibodies against canine respiratory viruses: prevalence among dogs of eastern Washington, Am J Vet Res (1974), pp. 853-855.

[22] B. Bibrack and W. Schaudinn, Studies on the occurrence of herpes infection in dogs in Western Germany with the help of a rapid neutralization test, Zbl Vet Med B 23 (1976), pp. 384-390.

[23] F.A.M. Rijsewijk, E.J. Luiten, F.J. Daus, R.W. van der Heijden and J.T. van Oirschot, Prevalence of antibodies against canine herpesvirus dogs in The Netherlands in 1997-1998, Vet Mircrobio/ 65 (1999), pp. 1-7.

[24] M.J. Reading and H.J. Field, A serological study of canine herpes virus-1 infection in the English dog population, Arch Virol 143 (1998), pp. 1477-1488. 
[25] M. Engels, M. Suter and B. Ruckstuhl, Detection of the canine herpesvirus in a case of whelp death, Schweiz Arch Tierheilkd 121 (1979), pp. 649-654.

[26] M. Ackermann, S. Grüninger, L. Bruckner, H.K. Müller, R. Zutter and U. Kihm, A simple ELISA for the estimation of antibodies to equine herpesvirus 1 and its application to the quality control of vaccines. In: Spear and Griffiths, Editors, Modern approaches to animal cell technology, ESACT, Butterworth (1988), pp. 613-627.

[27] M. Knuchel, M. Ackermann, H.K. Müller and U. Kihm, An ELISA for detection of antibodies against porcine epidemic diarrhoea virus based on the specific solubility of the viral surface glycoprotein, Vet Microbiol 32 (1992), pp. 117-134.

[28] R.G.D. Steel and J.H. Torrie, Principles and procedures of statistics a biomedical approach (2nd ed.), McGraw Hill, Singapore (1980) p. 633.

[29] M. Thrusfield, Veterinary epidemiology (3rd ed.), Blackwell Science Ltd., Oxford (2005) p. 584.

[30] I. Dohoo, W. Martin and H. Stryhn, Veterinary epidemiologic research, AVC Inc. Charlottetown, Prince Edward Island, Canada (2003).

[31] S.A. Reading and N.J. Dimmock, Neutralization of animal virus infectivity by antibody, Arch Virol 152 (2007), pp. 1047-1059. 